\title{
INTERACTION DE LA LUMIERE ET DE LA TEMPERATURE SUR LE TAUX DE CROISSANCE DE SCENEDESMUS CRASSUS
}

\author{
par M. GUERRI, L. BRUNEL et A. DAUTA ${ }^{1}$
}

Le taux de croissance de Scenedesmus crassus est décrit en fonction de l'intensité lumineuse. Différentes expressions de cette fonction sont testées. Les paramètres de l'équation résultante sont alors exprimés en fonction de la température. La comparaison des taux de croissance observés et simulés met en évidence la valeur prédictive du système élaboré.

\section{Growth rate of Scenedesmus crassus : light and temperature interactions.}

The growth rate of Scenedesmus crassus is described in function of various light intensities. Different expressions of this light function are tested. The parameters of resulting equations are defined as being functions of the temperature. The comparison between observed and predicted growth rates point out the predictive value of the developed model.

\section{1. - INTRODUCTION}

Les premières tentatives de modélisation (ex. Vollenweider 1969) visant à simuler une dynamique de population algale, avaient tendance à considérer le phytoplancton comme une unité homogène. Les études récentes (par ex. Tilman et Kilham 1976) font ressortir de réelles différences entre les espèces, et l'importance des constantes spécifiques, en tant que paramètre explicatif de la succession des espèces. Les modèles actuels (Di Toro et al. 1979, Canale et al. 1976, Bierman et al. 1976) sont déjà plus complexes et tendent à traiter chaque espèce séparemment selon ses réponses aux changements du milieu. Il est bien connu que dans les milieux naturels l'évolution de la biomasse d'algues planctoniques se ramène le plus souvent à la succession d'un nombre limité d'espèces dominantes qui constituent la fraction la plus productive du phytoplancton. Ces considérations nous ont conduits à l'étude, en laboratoire, d'une algue du genre Scenedesmus qui constitue une fraction importante du phytoplancton estival du Lot : Scenedesmus crassus Chod.

L'équation permettant d'écrire l'évolution d'une population A selon

1. Laboratoire d'Hydrobiologie ERA 702 du C.N.R.S., Université Paul-Sabatier, 118 , route de Narbonne, 31062 Toulouse Cedex. 
le temps t, peut s'exprimer par: $\mathrm{dA} / \mathrm{dt}=\mu \mathrm{A}$. Le taux de croissance $\mu$ de la population peut être décrit comme une fonction d'un taux maximal spécifique : $\mu$ max, affecté de facteurs de réduction qui sont des fonctions des variables climatiques : lumière-I, température- $\Theta$ (terme 1), et des éléments nutritifs : azote-N et phosphore-P (terme 2).

$$
\mu=\mu_{\mathrm{m}} \star[\operatorname{Red} \mathrm{I} \star \operatorname{Red} \Theta]_{\text {terme } 1}^{\star}[\operatorname{Red} \mathrm{N} \star \operatorname{Red} \mathrm{P}] \text { terme } 2
$$

où les symboles «* » représentent une combinaison des différents facteurs, combinaison qui n'est pas obligatoirement une multiplication.

Pour étudier l'influence du terme 1 , on peut éliminer l'impact du terme 2, en apportant les nutriments en excès. On définit alors le taux de oroissance en fonction de la lumière et de la température, première étape vers l'élaboration d'un modèle plus complexe, incluant l'influence des éléments nutritifs (Brunel et al., sous presse).

\section{2. - MATERIEL ET METHODES}

\subsection{Mode de culture}

La technique utilisée est celle des cultures en batch. La lumière est apportée par des tubes fluorescents, selon un cycle 15/9. Les cultures en phase exponentielle de croissance sont soumises à des combinaisons de température-lumière, variant respectivement entre 15 et $35^{\circ} \mathrm{C}$, et 0 et 40 Klux. Le milieu de culture est minéral (Brunel-Delclaux et Guerri 1980). L'apport en $\mathrm{CO} 2$ est réalisé par' bullage des cultures, les nutriments (N-NO3 et P-PO4) sont apportés en excès.

\subsection{Suivi des cultures}

La densité des cellules dans les cultures est estimée par une mesure de la densité optique à $678 \mathrm{~nm}$ (Sorakin 1973, Cloern 1976). La densité des cellules est suivie quotidiennement, et le taux de croissance est approché par la relation : $\mu=\log (\mathrm{A} 2 / \mathrm{A} 1)$ où $\mathrm{A} 1$ est la population à $\mathrm{t} 1$ et $\mathrm{A} 2$ la population à $\mathrm{t} 2$, avec $\mathrm{t} 2-\mathrm{t} 1=1$ jour.

\section{3. - RESULTATS}

\subsection{Résultats expérimentaux}

La fig. 1 rapporte le taux de croissance maximum associé à différentes intensités lumineuses à $25^{\circ} \mathrm{C}$. Des courbes analogues obtenues à diverses températures ont permis de définir un taux de croissance maximal ( $\mu$ max) et une intensité lumineuse optimale (Iopt). Ces résultats sont rassemblés dans la fig. 2 qui résume l'ensemble des taux de croissance obtenus pour les différentes combinaisons température- 


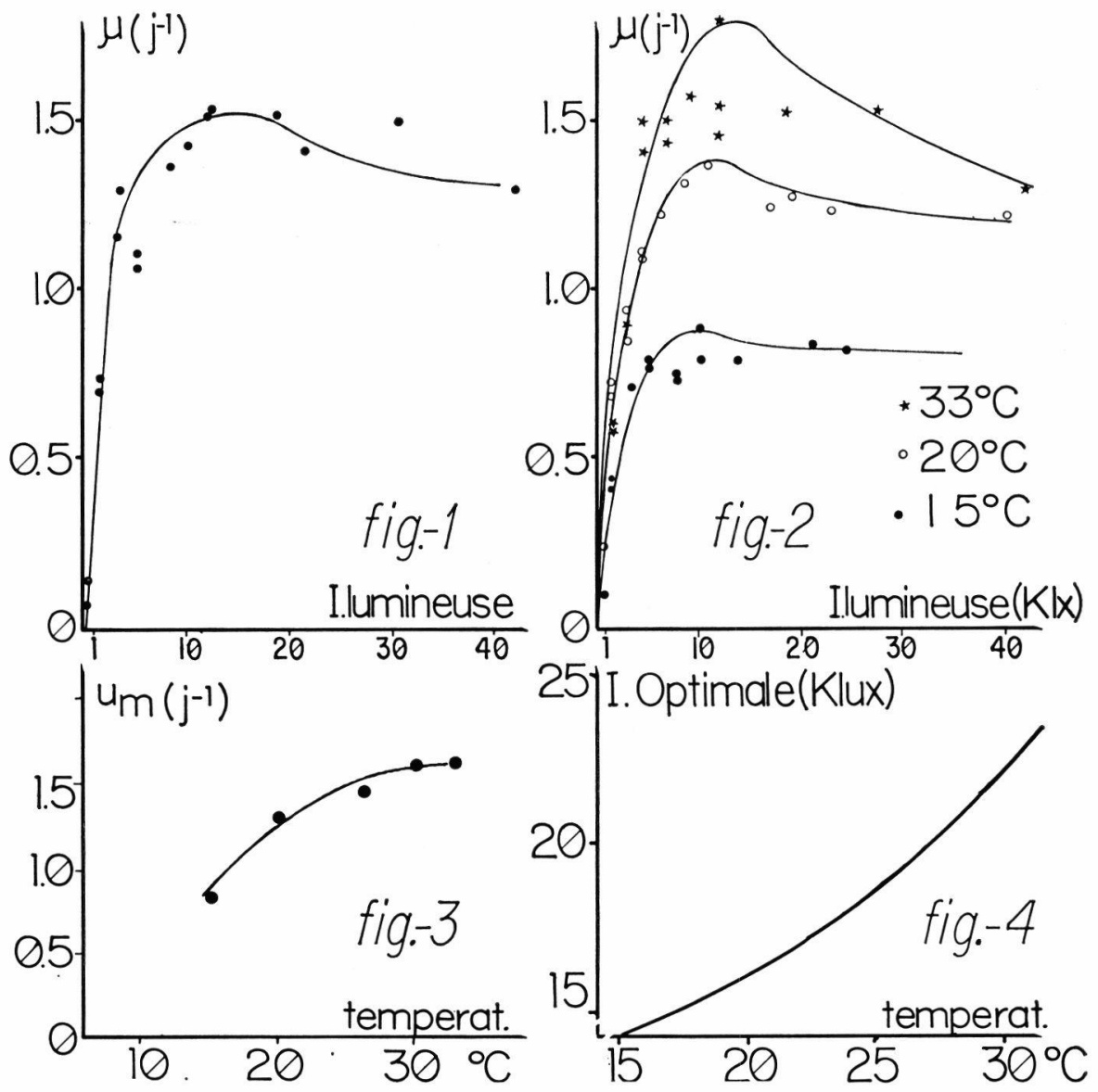

FIG. 1. - Taux de croissance de $\mu$ en fonction de l'intensité lumineuse I à $25^{\circ} \mathrm{C}$. Points expérimentaux ,nutriments non limitants.

Fig. 2. - Taux de croissance (points expérimentaux) pour des intensités lumineuses entre 0 et $40 \mathrm{Klux}$, aux températures de 15,20 et $33^{\circ} \mathrm{C}$.

Fig. 3. - Taux de croissance maximal observé, en fonction de la température.

FIG. 4. - Intensité de lumière saturante (I opt.) en fonction de la température.

lumière. Les fig. 3 et 4 donnent les variations de $\mu$ max et de Iopt en fonction de la température.

\subsection{Recherche d'une modélisation du taux de croissance}

Pour chacune des cinq températures étudiées, nous avons cherché à décrire la relation croissance-intensité lumineuse, en testant les trois grandes familles d'équations rencontrées dans la littérature. Ces 
expressions sont employées par divers auteurs, pour décrire soit un taux de croissance, soit un taux de photosynthèse en fonction de la lumière. Une analogie existe entre les deux, dans le cas où la population n'est pas limitée en nutriments, le facteur principal de croissance devenant alors le taux d'assimilation du carbone (Bougis 1974).

Six équations pouvant décrire les résultats ont été testées :

1) Equation de type Michaelis-Menten : $\mu=\mu \mathrm{m} . \mathrm{I} /(\mathrm{KI}+\mathrm{I})$, utilisée par exemple par Patten (1968), Chen-Orlob (1975), ou modifiée par Jorgensen (1976).

2) Equation de type Steele: $\mu=\mu \mathrm{m}$. a.I.exp (1-a.I.), utilisée par Steele (1962, Patten (1968), Larsen (1972), Lehman (1975), Cloern (1976).

3) Variante introduite par Vollenweider (1965): $\mu=\mu \mathrm{m}$. a.I.exp $(1-b I)$.

4) Equation de type Smith : $\mu=\mu \mathrm{m}$. a.I $/\left[1+(\mathrm{aI})^{2}\right]^{1 / 2}$, utilisée par Smith (1936), Talling (1957 a-b), Patten (1968), Fee (1969), avec des variantes introduites pour traduire une photo-inhibition, soit :

5) $\mu=\mu \mathrm{m} \cdot \mathrm{aI} /\left[1+(\mathrm{aI})^{2}\right]$.

6) $\mu=\mu \mathrm{m} \cdot \mathrm{aI} /\left[1+(\mathrm{aI})^{2}\right]^{1 / 2} \cdot\left[1+(\mathrm{bI})^{2}\right]_{1 / 2}$

L'adéquation des couples $(\mathrm{I}, \mu)$ aux équations précitées et à leurs variantes, a été calculée par la méthode des moindres carrés. Ces tests d'ajustement nous ont conduits à retenir une équation de type Smith :

$$
\mu_{0}=\mu \mathrm{m} . \mathrm{aI} /\left[1+(\mathrm{aI})^{2}\right]^{1 / 2}: \text { fig. } 5 .
$$

$\mu \mathrm{m}$ est le taux de croissance maximal que peut atteindre la population, à la température considérée, quand les conditions lumineuses et les conditions en nutriments sont optimales. Le début de la courbe peut être assimilé à une droite jusqu'à $I=1 / 2$ a, qui correspond à l'intensité seuil de la saturation pour la lumière, au-delà de laquelle la courbe s'infléchit. Le paramètre a renseigne également sur le degré d'adaptation de la population aux faibles intensités lumineuses.
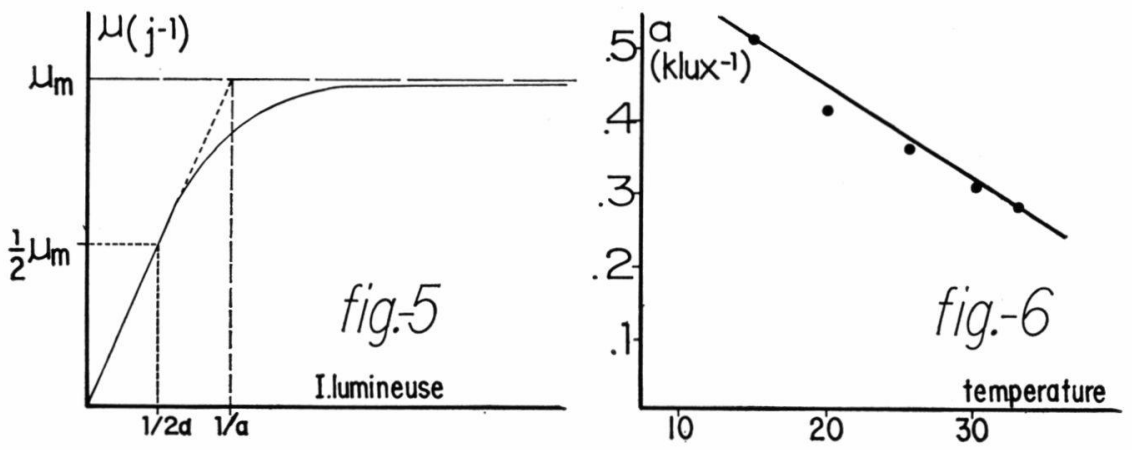

Fig. 5. - Equation de Smith. Courbe théorique.

Fig. 6. - Description du paramètre a, en fonction de la température. Points expérimentaux et courbe ajustée. 
La figure 6 représente l'ajustement de la courbe théorique, aux points observés pour $20^{\circ} \mathrm{C}$. Il apparaît que certains écarts avec la courbe théorique sont difficiles à systématiser, en particulier, la légère chute du taux de croissance pour une lumière supérieure à Iopt. Ce phénomène peut être interprété comme l'effet d'une photo-inhibition, compensé peu après par une adaptation des algues à la forte intensité lumineuse (le calcul du taux de croissance intégrant ces deux mécanismes successifs). Ces considérations nous ont amenés à conserver une modélisation de type Smith, peut-être moins fine, mais plus polyvalente.

\subsection{Calage du modèle}

La figure 2 fait clairement apparaître une interaction lumière-température sur le taux de croissance. Pour rendre compte de cette interaction et à l'exemple de Cloern (1976), nous avons décrit les paramètres $\mu \mathrm{m}$ et a en fonction de la température.

$-\mu \mathrm{m}(\Theta)$ s'ajuste à une portion de parabole (fig. 3) d'équation : $\mu \mathrm{m}(\Theta)=-.0026 \times \Theta^{2}+.1677 \times \Theta-\mathrm{I} .0797$

$\mu \mathrm{m}$ exprimé par jour.

- $\mathrm{a}(\Theta)$ s'ajuste à une portion de droite décroissante (fig. 7) selon une relation $: \mathrm{a}(\Theta)=-.0117 \Theta+.6638$

a exprimé en $\mathrm{Klux}^{-1}$.

$\mu$ peut alors être défini simplement en fonction de la température (fig. 8). De 1 à 15 Klux, l'influence de la lumière se retrouve dans l'étagement des courbes. Le sommet de chacune de ces courbes donne en ordonnée le plus fort taux de croissance possible à l'intensité lumineuse considérée, et en abcisse, la température optimale de croissance pour cette même intensité lumineuse. La température optimale augmente avec l'intensité lumineuse (fig. 9) jusqu'à un maximum de $32^{\circ} \mathrm{C}$.

Le taux de croissance correspondant, qui est de 1,62/jour, est alors le taux de croissance de la population en conditions optimales de lumière, température et nutriments.

La description du taux de croissance $\mu$ en fonction de l'intensité lumineuse I et de la température $\Theta$ peut être faite sous forme du système d'équation suivant :

$$
\begin{aligned}
& \mu=\mu \mathrm{m}(\Theta) \times \mathrm{a}(\Theta) \times \mathrm{I} /\left[1+(\mathrm{a}(\Theta) \mathrm{I})^{2}\right]^{1 / \mathrm{s}} \\
& \mu \mathrm{m} \Theta=.0026 \times \Theta^{2}+.1677 \times \Theta-1.0797 \\
& \mathrm{a}(\Theta)=.6638-.0117 \Theta
\end{aligned}
$$

avec $\mu$ et $\mu \mathrm{m}$ exprimés par jour, et a exprimé en $\mathrm{Klux}^{-1}$. Cette formulation a été testée en reportant pour 80 combinaisons de lumière et de température, le taux de croissance prédit et le taux de croissance observé (fig. 10). L'équation entre taux observés et prédits est satisfaisante, et montre ainsi la validité du modèle choisi. 

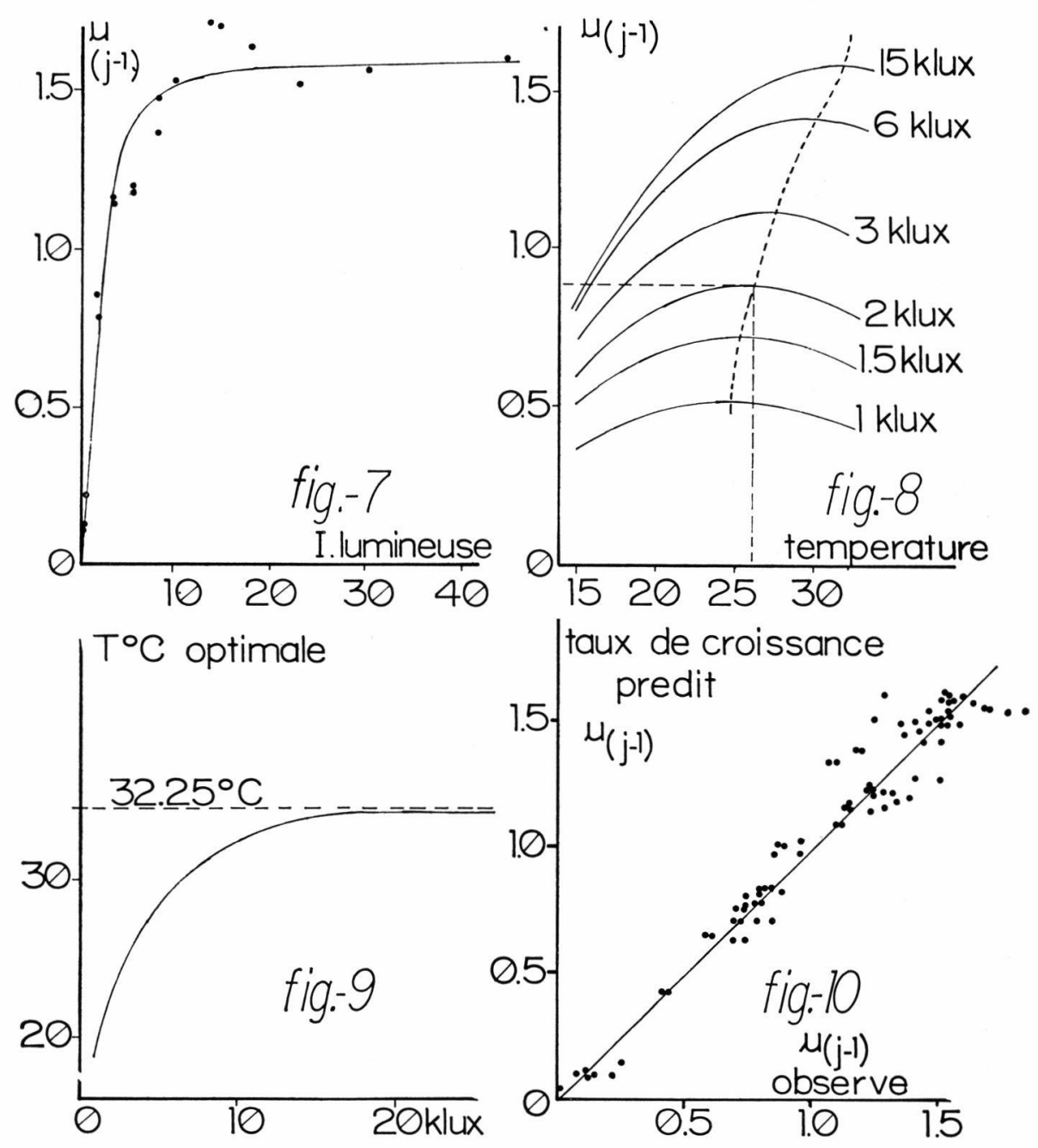

Fig. 7. - Ajustement du modèle réalisé, aux points expérimentaux pour $30^{\circ} \mathrm{C}$.

Fig. 8. - Taux de croissance en fonction de la température, pour différentes intensités lumineuses.

Fig. 9. - Relation entre températures optimales du taux de croissance, selon l'intensité lumineuse.

Fig. 10. - Taux de croissance prédits et taux de croissance observés.

\section{4. - DISCUSSION - CONCLUSION}

Parallèlement à la notion de température optimale, on peut s'intéresser à celle d'intensité lumineuse optimale : nos résultats font apparaître que ces deux paramètres sont interdépendants puisque (fig. 4) 
Iopt augmente régulièrement en fonction de la température, passant de 14 Klux pour $15^{\circ} \mathrm{C}$, à $25 \mathrm{Klux}$ pour $33^{\circ} \mathrm{C}$. La comparaison avec le milieu naturel montre que ces valeurs correspondent à celles de la partie supérieure de la zone trophogène d'eau. Ainsi dans le Lot, l'intensité lumineuse en surface atteint $100 \mathrm{Klux}$ dans la journée, mais du fait de la reflexion et de la turbidité, la lumière s'atténue rapidement avec la profondeur (Capblancq et Dauta 1978) : La profondeur limite de la zone euphotique, c'est-à-dire l'intensité des radiations actives pour la photosynthèse, égale à $1 \%$ de celle de surface, atteint 3,25 mètres en moyenne (variant de 0,7 à $5,3 \mathrm{~m}$ ). Ceci montre qu'en réalité l'intensité optimale ne correspondra souvent qu'à une couche d'eau superficielle très limitée. La fig. 9 met en évidence que dans un tel milieu naturel, où la température excède rarement $25^{\circ} \mathrm{C}$, Scenedesmus crassus est presque toujours en conditions suboptimales de température.

Une comparaison des résultats obtenus et des données bibliographiques montre que nos valeurs sont assez proches de celles données par différents auteurs : Lehman (1975) admet que pour les algues vertes $\mu \mathrm{m}$ est égal à $2,08 /$ jour pour Scenedesmus quadricauda. Mur et al. (1978) donnent pour le genre Scenedesmus $\mu \mathrm{m}=1,58 /$ jour. Jorgensen (1979) donne à $25^{\circ} \mathrm{C} \mu \mathrm{m}=2,02 /$ jour pour Scenedesmus quadricauda, et $\mu \mathrm{m}=1,52 /$ jour pour Scenedesmus obliquus, soit des taux très proches de ceux observés pour Scenedesmus crassus $(\mu \mathrm{m}=1,49 / \mathrm{jour} \grave{a}$ $25^{\circ} \mathrm{C}$ ).

L'intérêt du travail présenté réside dans l'interdépendance des paramètres mesurés. En effet, les modèles de production ou de croissance de phytoplancton se présentent souvent comme une combinaison ou un assemblage de paramètres d'origines diverses et de qualités différentes : le résultat se traduit généralement par une certaine fragilité et une faible fiabilité dans la prédiction. Ce mode de construction de modèle à partir de données disparates est remis en cause par Talling (1979) qui attire l'attention sur l'inadéquation des formulations multiplicatives, et met l'accent sur l'interdépendance des composantes utilisées dans le cadre de la modélisation.

\section{TRAVAUX CITÉS}

Bierman (V.J.) et Dolan (D. M.). 1976. - Mathematical modelling of phytoplankton dynamics in Saginaw Bay, Lake Huron. Proc. of the Conference on Environmental Modelling and Simulation, Cincinnati, April 1976, EPA Rep. 600/9-76-016: 773-779.

Brunel-Delclaux (L.), Guerri (M. M.). 1980. - Cinétiques d'assimilation et de croissance de Scenedesmus crassus. Etude expérimentale et modélisation. Thèse 3 cycle, Toulouse : $226 \mathrm{p}$.

Bougrs (P.). 1974. - Ecologie du phytoplancton marin. I. Le phytoplancton. Masson Ed., Paris : 196 p. 
Canale (R. P.), De Palma (L. M.) et Vogel (A. H.). 1976. - A plankton-based food web model for Lake Michigan. In R. P. Canale Ed., Modelling Biochemical Processes in Aquatic Ecosystems. Ann Arbor Sciences Pub., Ann Arbor, Michigan, 389 p. : 33-74.

Capblanco (J.) et Dauta (A.). 1978. - Phytoplancton et production primaire de la rivière Lot. Annls Limnol., 14 (1-2) : 85-112.

Chen (C. W.) et Orlob (G. T.). 1975. - Ecologic simulations for aquatic environments in: Systems analysis and simulations in ecology. III : 475-528. Ed. Patten (B. C.). Acad. Press. Inc.

CLOERN (J. E.). 1976. - Population dynamics of Cryptomonas ovata. A laboratory field and computer simulation study. Thèse P.H.D. Washington State Uni versity. Department of zoology : $102 \mathrm{p}$.

Di Toro (D. M.), O'Connor (D. J.), Thomann (R. V.) et Mancini (J. L.). 1975. Phytoplankton-zooplankton-nutrient interaction model for Western Lake Erié. In: Ed. Patten (B. C.), Systems Analysis and Simulation in Ecology. Tome III. Acad. Press, London, 558 p. : 423-474.

FEE (E. J.). 1969. - Numerical model for the estimation of P.S. production, integrated over time and depth in natural waters. Limnol. Oceanogr., 14 (6) : 906-911.

JoRGENSEN (S. E.). 1976. - An eutrophication model for a lake. Ecol. Modell., 2 : $147-165$.

JORGENSEN (S. E.). 1979. - Handbook of environmental Data and ecological para. meters. Ed. JoRGENSEN, Int. Soc. Ecol. Modell., $1162 \mathrm{p}$.

Larsen (D. P.), Mercier (H. T.) et Malveg (K. W.). 1972. - Modelling algal growth dynamics in Shagawa Lake, Minnesota, with comments concerning projected restoration of the lake. In: Modelling the eutrophication process: 1533. Ed. Middlebrooks, FalKenderg, Maloney. Ann Arbor publishers.

Lehman (J. T.), BotKin (D. B.) et Likens (G. E.). 1975. - The assumptions and rationales of a computer model of phytoplankton population dynamics. Limnol. Oceanogr., 20 (3) : 343-364.

MUR (L. R.), Gons (H.J.) et VANLIERe (L.). 1978. - Competition of the green alga Scenedesmus and the blue-green algal Oscillatoria. Mitt. Int. Ver. Limnol., $21: 473-479$.

Patten (B. C.). 1968. - Mathematical models of plankton production. Int. Rev. Ges. Hydrobiol., 53 (3) : 357-408.

Sorokin (C.). 1973. - Dry weight, packed cell volume and optical density. In : Handbook of phycological methods: 321-343. J. R. STEIN Ed. Cambridge.

SMITH (E. L.). 1936. - Photosynthesis in relation to light and carbon dioxide. Proc. Nat. Acad. Sc. Wash., 22 : 504-511.

STEELE (J. H.). 1962. - Environmental control of photosynthesis in the sea. Limnol. Oceanog., $7:$ 137-150.

Talling (J. F.). 1957 a. - Photosynthetic characteristics of some freshwater plankton diatoms in relation to underwater radiation. New Phytol., 56: 29-50.

TALLING (J. F.). $1957 \mathrm{~b}$. - The phytoplankton population as a compound photosynthetic system. New Phytol., $56: 133-149$.

Talling (J. F.). 1979. - Factor interactions and implications for the prediction of lake metabolism. Arch. Hydrobiol., $13: 96-109$.

Tilman (D.) et KILham (S. S.). 1976. - Phosphate and silicate growth and uptake kinetics of the diatoms Asterionnella formosa and Cyclotella meneghiniana in batch and semi-continuous culture. J. Phycol., 12 (4) : 375-383.

Vollenweider (R. A.). 1965. - Calculation models of photosynthesis : depth curves and some implications regarding day rate estimates in primary production measurements. Mem. Ist. Ital. Idrobiol., 18 (suppl.) : 425-457.

VollenWEIder (R.A.). 1969. - Möglichkeiten und Grenzen elementarer Modelle des Stoffbilanz von Seen. Arch. Hydrobiol., $66: 1-36$. 